\title{
A Novel Anti-HER2 Bispecific Antibody With Potent Tumor Inhibitory Effects In Vitro and In Vivo
}

\begin{abstract}
Mehdi Mohammadi ${ }^{1}$, Mahmood Jeddi-Tehrani ${ }^{3}$, Forough Golsaz-Shirazi ${ }^{1}$, Mohammad Arjmand ${ }^{2}$, Tannaz Bahadori ${ }^{1}$, Mohammad Ali Judaki ${ }^{1}$, Fariba Shiravi ${ }^{1}$, Hengameh Ahmadi Zare ${ }^{3}$, Farzaneh Notash Haghighat ${ }^{3}$, Maryam Mobini ${ }^{1}$, Mohammad Mehdi Amiri ${ }^{1 *}$ and Fazel Shokri ${ }^{1 *}$
\end{abstract}

\begin{abstract}
${ }^{1}$ Department of Immunology, School of Public Health, Tehran University of Medical Sciences, Tehran, Iran, ${ }^{2}$ Department of Biochemistry, Pasteur Institute of Iran, Tehran, Iran, ${ }^{3}$ Monoclonal Antibody Research Center, Avicenna Research Institute, Academic Center for Education, Culture and Research (ACECR), Tehran, Iran
\end{abstract}

OPEN ACCESS

Edited by: Giovanna Schiavoni, National Institute of Health (ISS), Italy

Reviewed by: Junpeng Qi, The Scripps Research Institute, United States Piero Pileri,

Toscana Life Sciences, Italy

${ }^{*}$ Correspondence: Fazel Shokri

Fshokri@tums.ac.ir Mohammad Mehdi Amir m_amiri@tums.ac.ir

Specialty section: This article was submitted to Cancer Immunity and Immunotherapy, a section of the journa Frontiers in Immunology

Received: 31 August 2020 Accepted: 23 December 2020 Published: 17 February 2021

Citation:

Mohammadi M, Jeddi-Tehrani M, Golsaz-Shirazi F, Arimand M, Bahadori T, Judaki MA, Shiravi F, Zare HA, Haghighat FN, Mobini M, Amiri MM and Shokri F (2021) A Novel Anti-HER2 Bispecific Antibody With

Potent Tumor Inhibitory Effects In Vitro and In Vivo.

Front. Immunol. 11:600883. doi: 10.3389/fimmu.2020.600883
Overexpression of HER2 has been reported in many types of cancer, making it a perfect candidate for targeted immunotherapy. The combination of two FDA approved monoclonal antibodies (mAbs), trastuzumab and pertuzumab, has more robust anti-tumor activity in patients with HER2-overexpressing breast cancer. We recently produced a new humanized anti-HER2 mAb, hersintuzumab, which recognizes a different epitope than trastuzumab and pertuzumab on HER2. This mAb, in combination with trastuzumab, exhibits more potent anti-tumor activity than each parental mAb alone. Here we have developed a novel bispecific anti-HER2 antibody (BsAb) designated as trasintuzumab, composed of trastuzumab and hersintuzumab, using dual variable domain immunoglobulin (DVD-lg) technology. Both variable domains of trasintuzumab are fully functional and have similar affinities to the parental mAbs and are also able to bind to natural HER2 on the surface of several HER2expressing cell lines. Trasintuzumab was found to inhibit the growth of different types of tumor cell lines through suppression of the AKT and ERK signaling pathways as efficiently as the combination of the parental mAbs. It also induced tumor regression as potently as the combination of the two mAbs in nude mice bearing ovarian and gastric cancer xenografts. Our data suggest that trasintuzumab may be a promising BsAb therapeutic candidate for the treatment of HER2-overexpressing cancers.

Keywords: HER2, DVD-Ig, monoclonal antibody, bispecific antibody, cancer immunotherapy

\section{INTRODUCTION}

The human HER (human epidermal growth factor receptor) family includes HER1 (ErbB1, also known as EGFR), HER2 (ErbB2, c-erbB2, or HER2/neu), HER3 (ErbB3), and HER4 (ErbB4) molecules. These receptors are structurally similar and their hetero/homo dimerizations play important roles in cell differentiation, growth, and survival (1). Among this family, HER2 is studied more extensively. It is a $185 \mathrm{kDa}$ protein with four extracellular domains (ECD) and an intracellular tyrosine kinase domain, which mainly signals through phosphatidylinositol-3 kinase (PI3K) and mitogen-activated protein kinase (MAPK) signaling pathways (2). There is no identified ligand for 
HER2 and it forms heterodimers with other members of the HER family to activate downstream signaling pathways. Overexpression of HER2 is associated with disease prognosis and has been observed in approximately $25 \%-30 \%$ of breast cancers, $6 \%-35 \%$ of gastric cancers, $9 \%-32 \%$ of ovarian cancers, and $25 \%-78 \%$ of prostate cancers (3).

Since the discovery of the HER2 role in tumor progression, extensive efforts have been made to produce monoclonal antibodies (mAbs) against this target. Trastuzumab is the first mAb against domain IV of HER2 (4), which was approved by the FDA for breast cancer in 1998 and for metastatic stomach cancer in 2010. Pertuzumab is another mAb that targets domain II of HER2 (5). Since trastuzumab and pertuzumab bind to different domains of HER2 and are non-competitive, their combination therapy showed synergistic effects (6) and was later approved for treatment of metastatic breast cancer in 2012. Based on the synergistic effects of trastuzumab and pertuzumab against different epitopes of HER2, efforts have been made globally to develop novel antibodies for combination therapies. Bong-Kook and colleagues developed a humanized anti HER2 mAb, 1E11, that showed synergistic effects in combination with trastuzumab in gastric cancer cell lines (7). In another study, a mixture of three non-overlapping anti-HER2 antibodies showed a stronger anti-tumor activity than the combination of trastuzumab with pertuzumab in vitro and in vivo (8). It was also reported that a $\mathrm{mAb}$ targeting ErbB2 domain III provided synergy with either of trastuzumab or pertuzumab for inhibition of HER2 heterodimerization and cell signaling (9). GB235, another antiHER2 mAb, in combination with trastuzumab inhibited tumor growth in the NCI-N87 gastric xenograft tumor model (10). There are other studies that reported similar results using different combinations of anti-HER2 mAbs (11).

Recently, we produced a humanized mAb, hersintuzumab, which targets domains I-II of human HER2, a distinct epitope from those recognized by trastuzumab and pertuzumab (12). Hersintuzumab induced growth inhibition, G1 cell cycle arrest, and cell signaling inhibition in HER2 expressing tumor cell lines (12). This mAb displayed significant synergistic inhibitory effects in combination with trastuzumab.

Bispecific antibodies (BsAbs) could be used to target two epitopes on the same or on distinct molecules instead of using two mAbs. So far, three BsAbs were approved in the USA and Europe (13), and many promising BsAbs are in the pipeline, targeting either two non-overlapping epitopes on HER2 (14-20) or HER2 together with other molecules such as HER3 $(21,22)$, CD3 (23-26), PD-L1 (27), 4-1BB (28), or CD63 (29).

Various molecular formats of BsAbs have been developed (30). Dual variable domain immunoglobulin (DVD-Ig) is composed of variable domains of two pre-existing mAbs via naturally occurring linkers, which yields a tetravalent IgG-like $\mathrm{BsAb}$ (31). In addition to a wide range of DVD-Igs directed against tumors $(14,16,19,32-34)$, several DVD-Igs targeting a variety of specificities such as TNF- $\alpha$, IL-17A (35), IL-1 $\alpha$, and IL-1 $\beta$ (36) for treatment of autoimmune diseases, VEGF and PDGF for treatment of age-related macular degeneration (37), gp41 and gp120 for treatment of AIDS (38), TGF- $\beta$ and fibronectin extra domain A (FN-EDA) for treatment of fibrotic kidneys (39), and amyloid-beta and transferrin receptor for treatment of Alzheimer's disease (40) have been constructed and characterized recently. Some of these DVD-Igs are currently undergoing clinical trials (41-43).

In this study we generated and characterized a BsAb against HER2 in DVD-Ig platform, using variable domains of trastuzumab and hersintuzumab.

\section{MATERIALS AND METHODS}

\section{Cell Lines}

Human breast cancer cell lines BT-474, MCF-7, JIMT-1, HCC1954, human ovarian cancer cell line SKOV-3, human gastric cancer cell line NCI-N87 and CHO-K1 cells were obtained from the National Cell Bank of Iran (Pasture Institute of Iran, Tehran, Iran). All cell lines were cultured in RPMI 1640 medium (Gibco, Grand Island, NE, USA) supplemented with $100 \mathrm{U} / \mathrm{mL}$ penicillin, $100 \mu \mathrm{g} / \mathrm{mL}$ streptomycin (Gibco), and 10\% fetal bovine serum (Gibco). BT-474 was cultured in $20 \%$ fetal bovine serum (Gibco) and also supplemented with $10 \mu \mathrm{g} / \mathrm{mL}$ human insulin (Exir Co., Boroojerd, Iran). 3T3-L1 was obtained from Iranian Biological Resource Center (Tehran, Iran) and was cultured in DMEM medium (Gibco) supplemented with $100 \mathrm{U} /$ $\mathrm{mL}$ penicillin, $100 \mu \mathrm{g} / \mathrm{mL}$ streptomycin (Gibco), and 20\% fetal bovine serum (Gibco).

\section{Design, Expression, Purification, and Structural Characterization of DVD-Igs}

Two forms of anti-HER2 DVD-Igs, based on the proximity of variable domains of trastuzumab or hersintuzumab to N-terminal of the heavy and light chains of DVD-Ig, were designed as described previously (31). Briefly, the VL and VH sequences of hersintuzumab (12) and VL (GenBank: GM685466.1) and VH (GenBank: GM685464.1) sequences of trastuzumab were attached with two linkers ASTKGPSVFPLAP and TVAAPSVFIFPP, respectively, and then joined to human IgG1 heavy and CK light chain constant domains. These linkers are basically the same as the natural linkers present between the constant and variable domains of the heavy and light chains of an IgG molecule, providing a flexible conformation to both domains. These domains have been widely used in various DVD-Igs $(33,34,44,45)$. The genes of heavy and light chains of DVD-Igs were synthesized (Biomatik Co., Cambridge, ON, Canada) and cloned to a homemade dualpromoter expression plasmid that contains glutamine synthetase (GS) gene as a selection marker. The plasmid was then transfected to $\mathrm{CHO}-\mathrm{K} 1$ cells by Lipofectamin 3000 reagent (Lifetechnology, USA) and stable clones were selected in the presence of methionine sulfoximine (MSX). The final selected highproducing clone was cultured in serum free medium and recombinant antibodies were purified with protein A affinity chromatography (GE Healthcare, USA). The purified DVD-Igs were run on $8 \%$ SDS-PAGE under reducing and non-reducing conditions. After electrophoresis, the gel was stained with Coomassie brilliant blue. 


\section{Dual Specific Binding of DVD-Igs to Recombinant and Native Forms of HER2}

For specific binding of each domain of DVD-Igs, recombinant full HER2-extracellular domain (ECD) and its subdomains, I+II and III+IV, which had been produced in our previous study (46), were coated in ELISA plate for 1.5 hours at $37^{\circ} \mathrm{C}$. After 1 hour blocking with PBS containing $0.05 \%$ tween 20 (PBS-T) and 3\% skim milk (Merck, Darmstadt, Gemany) at $37^{\circ} \mathrm{C}$, different concentrations of DVD-Igs or parental mAbs were added and incubated for 1 hour at $37^{\circ} \mathrm{C}$ and then HRP-conjugated rabbit anti human Ig (Sinabiotech Co., Tehran, Iran) was added and incubated for another 1 hour. After addition of TMB substrate (Pishtazteb Co., Tehran, Iran) at room temperature, the reaction was stopped with HCL $1 \mathrm{~N}$, and finally ODs were measured by an ELISA plate reader (Beckman Coulter, Brea, California, USA) at $450 / 630 \mathrm{~nm}$.

Total IgG concentration was determined by an immunoglobulinspecific ELISA. Briefly, sheep anti-human Ig polyclonal antibody (SinaBiotech) was coated in 96 well-ELISA plate and blocked as previously described. Different concentrations of antibodies were then added and after incubation for 1 hour at $37^{\circ} \mathrm{C}$ and washing, HRP-sheep anti-human Ig polyclonal antibody (SinaBiotech) was added and the experiment proceeded as indicated above.

For competitive ELISA assay, recombinant full HER2-ECD or its subdomains, I+II and III+IV, were coated in ELISA plate for 1.5 hours at $37^{\circ} \mathrm{C}$. After 1 hour blocking with PBS-T and 3\% skim milk at $37^{\circ} \mathrm{C}$, serial dilutions of DVD-Igs or parental mAbs were mixed with HRP-labeled trastuzumab or HRP-labeled hersintuzumab and incubated for an additional 1 hour at $37^{\circ} \mathrm{C}$. After addition of TMB substrate at room temperature, the reaction was stopped with HCL $1 \mathrm{~N}$, and finally ODs were measured by an ELISA plate reader at $450 / 630 \mathrm{~nm}$. The IC50 values of competitors were calculated using a four-variable algorithm.

For flow cytometry, different cell lines with various levels of HER2 expression were trypsinized and after twice washing with PBS, incubated with $100 \mu \mathrm{l}$ of trastuzumab, hersintuzumab, or DVD-Igs at final concentration of $5 \mu \mathrm{g} / \mathrm{ml}$ on ice for 45 minutes. A chimeric anti-hepatitis B mAb harboring human IgG1/ $\kappa$ was used as isotype control (47). Cells were then washed and incubated with FITC-labeled sheep anti-human Ig antibody (Sinabiotech) on ice for additional 45 minutes, and after washing, cells were scanned by a flow cytometer (Partec, Nuremberg, Germany), and data were analyzed using the FlowJo v10 software.

For flow cytometry competitive binding assay, $1 \times 10^{6}$ SKOV -3 cells were incubated with increasing concentrations of DVD-Igs, trastuzumab, or hersintuzumab and incubated for 45 minutes on ice. Then, FITC labeled trastuzumab or hersintuzumab (prepared in our laboratory) were added to the tubes and incubated on ice for another 45 minutes. After twice washing with PBS, cells were analyzed by flow cytometer. The IC50 values of competitors were calculated using a four-variable algorithm.

\section{Affinity Measurement}

Binding affinity of DVD-Igs was measured with ELISA, as described previously (12). Briefly, serial concentrations (1200-
$75 \mathrm{ng} / \mathrm{mL}$ ) of recombinant subdomains I+II, III+IV, or full HER2-ECD were coated in ELISA plate for 1.5 hours at $37^{\circ} \mathrm{C}$. After 1 hour blocking with PBS-T containing 3\% skim milk at $37^{\circ} \mathrm{C}$, serial concentrations $(1000-15.6 \mathrm{ng} / \mathrm{mL})$ of DVD-Igs were added to the wells and incubated for 1 hour at $37^{\circ} \mathrm{C}$. Then, HRPconjugated rabbit anti-human Ig antibody was added and after 1 hour incubation at $37^{\circ} \mathrm{C}$ and washing twice with PBS-T, TMB substrate was added and after 5-10 min incubation at room temperature, the reaction was stopped with HCL 1N. Finally, the plate was read with plate reader at $450 / 630 \mathrm{~nm}$. ODs were plotted against logarithmic values of antibody concentration and the antibody concentration giving $50 \%$ of the maximum absorbance value $([\mathrm{Ab}] \mathrm{t})$ at a particular antigen coating concentration was chosen for the affinity measurement using the formula $K_{D}=1 / 2$ $\left(2\left[\mathrm{Ab} \mathrm{b}^{\prime}\right] \mathrm{t}-[\mathrm{Ab}] \mathrm{t}\right)$. [A $\left.\mathrm{Ab}^{\prime}\right] \mathrm{t}$ and $[\mathrm{Ab}] \mathrm{t}$ represents the antibody concentrations resulting in $50 \%$ of maximum absorbance value at two consecutive concentrations of coated antigen where $[\mathrm{Ag}]$ $=2\left[\mathrm{Ag}^{\prime}\right]$. The mean of such calculations for three nonoverlapping antigen concentrations was taken as the final $\mathrm{K}_{\mathrm{D}}$ value.

\section{Proliferation Inhibition of Various Cancer Cell Lines by DVD-Igs}

$6 \times 10^{3}$ BT-474, $2 \times 10^{3}$ JIMT-1, $3.5 \times 10^{3}$ HCC- $1954,1.5 \times 10^{3}$ SKOV-3, and $11 \times 10^{3}$ NCI-N87 cells were seeded in flatbottomed 96-well plates and incubated overnight in a humidified atmosphere containing $5 \% \mathrm{CO} 2$ at $37^{\circ} \mathrm{C}$. DVD-Igs, hersintuzumab, trastuzumab $(20,1,0.2$, and $0.04 \mu \mathrm{g} / \mathrm{ml})$ or combination of hersintuzumab and trastuzumab $(10,0.5,0.1$, and $0.02 \mu \mathrm{g} / \mathrm{ml}$ of each) were added to the wells, and after 3 days of incubation, $1 \mu \mathrm{Ci} 3 \mathrm{H}$-thymidine (PerkinElmer, MA, USA) was added to each well and incubated for an additional 20 hours. The cells were then harvested and transferred to the scintillation fluid and the amount of $3 \mathrm{H}$-thymidine uptake was measured by a $\beta$ counter (Wallac 1410 Liquid Scintillation Counter, Pharmacia, Sweden). All experiments were performed at three independent times in triplicates. The proliferation inhibition rate was calculated with the use of following formula:

Proliferation inhibition $(\%)=([$ Counts per minute $(\mathrm{CPM})$ without antibody - CPM with antibody]/CPM without antibody) $\times 100$.

\section{Effect of DVD-Igs on Downstream Cell Signaling Pathways}

Cell signaling studies were done as described previously (48). Briefly, BT-474, NCI-N87, SKOV-3, HCC-1954, JIMT-1, and MCF-7 cells were seeded in T-25 flasks and after an overnight incubation at $37^{\circ} \mathrm{C}$ in a humidified atmosphere containing $5 \%$ $\mathrm{CO} 2$, trastuzumab plus hersintuzumab or $\mathrm{BiHT}$ were added to the flask at a final concentration of $20 \mu \mathrm{g} / \mathrm{ml}$ and excipient was used as control. After 24 hours of incubation at $37^{\circ} \mathrm{C}$ and $5 \%$ $\mathrm{CO} 2$ atmosphere, cells were then trypsinized and lysed with $\mathrm{M}$ PER reagent (Thermo Fisher Scientific, Waltham, Massachusetts, USA) containing $\mathrm{Halt}^{\mathrm{TM}}$ protease and phosphatase inhibitor (Thermo Fisher Scientific). Total protein content of cell lysates 
A

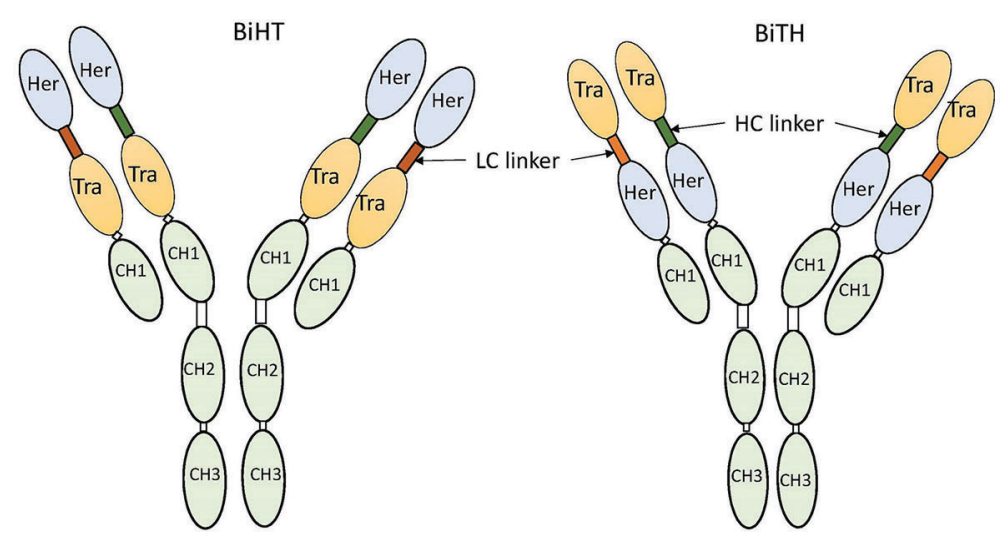

B

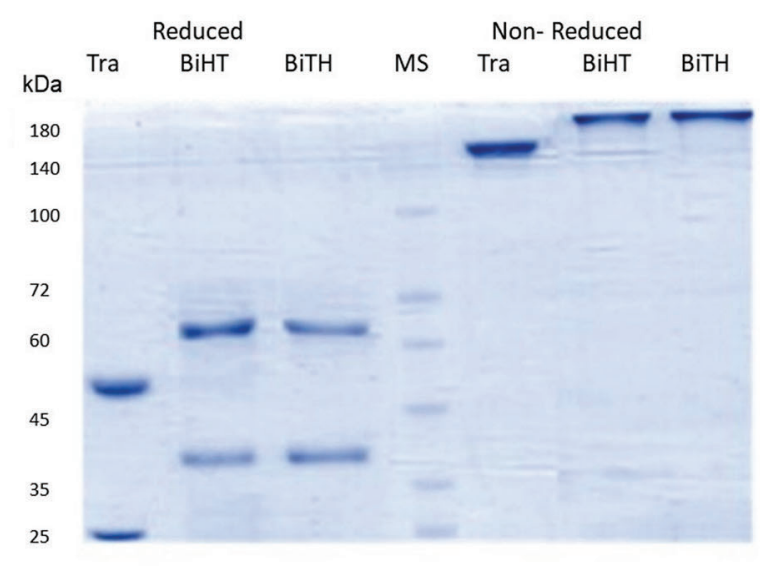

FIGURE 1 | Structural characterization of anti-HER2 DVD-Igs. (A) Schematic presentation of BiHT and BiTH design. (B) SDS-PAGE analysis of trastuzumab, BiHT and BiTH under reducing (left) and non-reducing (right) conditions. Tra, trastuzumab; Her, hersintuzumab; HC, heavy chain; LC, light chain; MS, molecular size.

was normalized using BCA assay kit (Thermo Fisher Scientific) and were subjected to $10 \%$ SDS-PAGE, and after transferring to PVDF membrane, immunoblotting was done using antibodies against phosphorylated AKT, phosphorylated ERK, AKT, ERK, HER2, and $\beta$-actin as primary antibodies and HRP-conjugated anti-rabbit antibody (Cell signaling Technology, Danvers, Massachusetts, USA) as secondary antibody. These experiments were done once for all cell lines. Finally, protein bands were visualized with enhanced chemiluminescence prime kit (ECL, GE Healthcare, Uppsala, Sweden), scanned, and analyzed with ImageJ software. Band density was calculated using the following formula:

Band density $=\left(\frac{\text { band density of mAb }}{\text { band density of its corresponding } \beta \text { Actin }}\right) /$

$$
\left(\frac{\text { band density of Control (excipient) }}{\text { band density of its corresponding } \beta \text { Actin }}\right)
$$

\section{Antibody-Dependent Cell-Mediated Cytotoxicity (ADCC)}

A standard lactate dehydrogenase $(\mathrm{LDH})$ release assay was performed to analyze ADCC. Normal human peripheral blood mononuclear cells (PBMCs) and SKOV-3 cells were used as effector and target cells respectively. Target cells were seeded in U-bottom 96-well plates at 10,000 cells/well and opsonized with serial dilutions of DVD-Igs and parental mAbs for 30 minutes. A chimeric anti-hepatitis $\mathrm{B} \mathrm{mAb}$ harboring human $\mathrm{IgG} 1 / \kappa$ was used as isotype negative control (47). Then, PBMCs were added at 500,000/well and incubated in a humidified atmosphere containing $5 \% \mathrm{CO} 2$ at $37^{\circ} \mathrm{C}$ for an additional $5 \mathrm{~h}$. Then, $2 \%$ Triton-X 100 was used for maximum release of the LDH from target cells. The LDH released from cells in the culture supernatant was determined using a cytotoxicity assay kit (Promega, Madison, WI, USA). The percentage of cytotoxicity was calculated by the following formula:

Cytotoxicity \% = (Experimental release - Effector cells spontaneous release - Target cells spontaneous release)/ 
(Target cells maximum release -Target cells spontaneous release) $\times 100$.

\section{Pharmacokinetics of BiHT}

To determine pharmacokinetic properties, a single dose of $10 \mathrm{mg} /$ $\mathrm{kg}$ of BiHT, hersintuzumab, or trastuzumab were injected intraperitoneally (IP) to 6-week-old female BALB/c mice. Trastuzumab was also injected intravenously (IV) as a control. Blood samples were taken from the lateral tail vein at $30 \mathrm{~min}, 2 \mathrm{~h}$, and 1, 2, 7, 14, 21 days after administration and sera were collected and stored at $-20^{\circ} \mathrm{C}$. The antibody levels were measured by a sandwich ELISA. Briefly, mouse mAb specific to human IgG1 (Sinabiotech) were coated overnight in an ELISA plate at $4^{\circ} \mathrm{C}$ and after 1 hour blocking with PBS-T containing $3 \%$ skim milk at $37^{\circ} \mathrm{C}$, serial dilutions of mouse sera were added into the wells. Then, mouse-adsorbed HRP-conjugated rabbit anti-human Ig (Sinabiotech) were added and after 1 hour of incubation at $37^{\circ} \mathrm{C}$ and three times washing with PBS-T, TMB substrate was added and the reaction was then stopped with $1 \mathrm{~N} \mathrm{HCL}$. Finally, the ODs were measured with a plate reader at 450/630 nm. Pharmacokinetic parameters were calculated by a noncompartmental analysis using Phoenix Winnonlin (31).

\section{Tumor Growth Inhibition In Vivo}

Two HER2 over-expressing cell lines were employed for xenograft study. NCI-N87 cells $\left(5 \times 10^{6}\right.$ in Matrigel; Corning, Life Sciences, Corning, NJ) and SKOV-3 cells $\left(5 \times 10^{6}\right.$ in PBS) were implanted subcutaneously into the right flank of female $\mathrm{BALB} / \mathrm{c}$ nude mice. When the tumor volume reached about $100-$ $150 \mathrm{~mm}^{3}$, the mice were randomly divided in three groups of 6 mice. DVD-Ig (10mg/kg), Trastuzumab plus Hersintuzumab (5 $\mathrm{mg} / \mathrm{kg}$ of each), or PBS were intraperitoneally injected once a week for 6 consecutive weeks. Tumor sizes were measured three times a week and the tumor volumes were calculated by the formula: volume $=$ length $\times(\text { width })^{2} / 2$. All animal experiments were performed according to the permission granted by the Ethics Committee of Tehran University of Medical Sciences (IR NIMAD REC 1396 060).

\section{Statistics}

The results were analyzed with One-Way ANOVA test using SPSS software (version 20, IBM SPSS statistics data editor). Differences between groups were significant at $\mathrm{p}$ values less than $0.05(\mathrm{p}<0.05)$. The results are presented as mean \pm standard error of the mean (SEM).

\section{RESULTS}

\section{Design, Construction, Expression, and Characterization of Anti-HER2 DVD-Igs}

Two DVD-Igs (BiHT and BiTH) were engineered using variable domains of trastuzumab and hersintuzumab, which are joined together in tandem with different orientations (Figure 1A). Both DVD-Igs were expressed in conjunction with human IgG1 and
CK constant domains. The structure of DVD-Igs were analyzed by SDS-PAGE, and the results showed similar patterns for both DVD-Igs with a monomeric form $(200 \mathrm{kDa})$ under nonreducing and two monomeric heavy $(\sim 65 \mathrm{kDa})$ and light $(\sim 40 \mathrm{kDa})$ chains under reducing conditions (Figure 1B).

The constructs were transfected in CHO cells, and the BsAbs were screened in culture supernatant by ELISA. Immunoglobulinspecific ELISA was used to determine the concentration of each antibody (Figure 2A), and the same molar concentration of DVDIgs and mAbs were used in all experiments. Dual specific binding and affinity of DVD-Igs to subdomain I+II (hersintuzumab epitope), subdomain III+IV (trastuzumab epitope), and full ECD of human HER2 were assessed by a HER2-specific ELISA. The results demonstrated that both DVD-Igs were able to bind specifically to their epitopes. The binding profiles of both BiHT and BiTH to full ECD and subdomain III+IV at different concentrations were quite similar to those of the parental mAbs (Figure 2B). However, while reactivity of BiHT to subdomain I+II was similar to hersintuzumab, reactivity of BiTH to subdomain I + II was notably weaker than that of the parental hersintuzumab (Figures 2C, D).

The affinity constant of each BsAb to HER2-ECD was similar to the mean of affinity constants of the parental mAbs (Table 1). The affinity constant of BiHT to DI+II and DIII+IV (i.e., the target of its inner and outer variable domains, respectively) have decreased 2-3 times, as compared to the corresponding parental mAbs (Table 1). However, while the affinity constant of BiTH to DIII+IV (i.e., the target of its outer variable domain) was similar to that of trastuzumab, its affinity to DI+II (i.e., the target of its inner variable domain) has decreased 11 times as compared to the corresponding parental $\mathrm{mAb}$, hersintuzumab (Table 1). In other words, the variable domain of trastuzumab is more compatible with the inner orientation of DVD-Ig, as compared to hersintuzumab.

Competitive binding assays were used to assess the relative binding affinity of BiHT and BiTH for their epitopes expressed on recombinant HER2-ECD, subdomain I+II, or subdomain III $+\mathrm{IV}$, using ELISA (Figures 3A-D) and flow cytometry (Figures 3E, F). The results showed that IC50 values of BiHT for recombinant HER2-ECD (Figure 3A), subdomain III+IV (Figure 3C) and native HER2 (Figures 3E, F) were similar to the parental mAbs. Despite the slight decrease in the IC50 values in competitive ELISA (Figures 3B, D), BiTH retained the full binding activity for native HER2 in competitive flow cytometry (Figures 3E, F).

In order to determine the binding profile of DVD-Igs to the HER2-overexpressing tumor cells, flow cytometry analysis was conducted. Both DVD-Igs could detect HER2 on the surface of different cancer cell lines similar to trastuzumab and hersintuzumab with regards to percent of positive cells and mean fluorescence intensity (Figure 4). Taking together, results of binding assays demonstrate that BiHT retains full binding activity to both of the corresponding epitopes in comparison to the parental mAbs. However, the binding activity of BiTH is decreased as indicated by the competitive ELISA results (Figures 3B, D). 
A

Immunoglobulin-specific ELISA

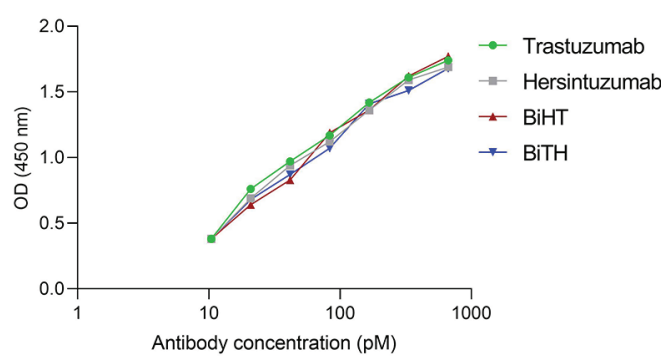

C

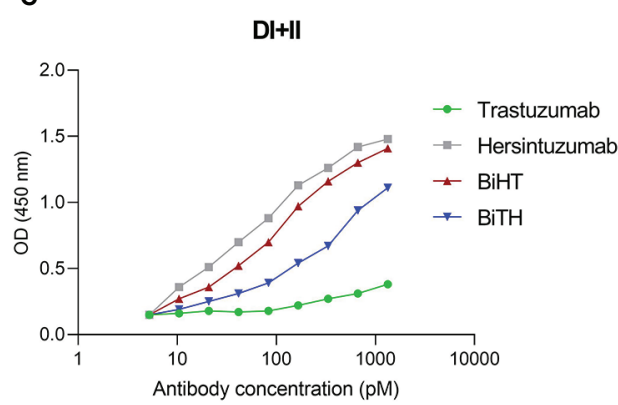

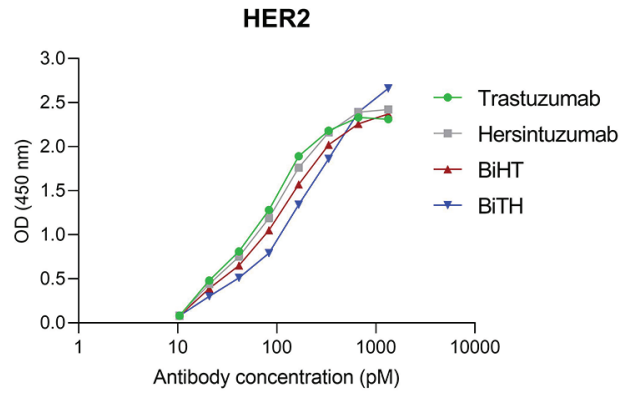

D

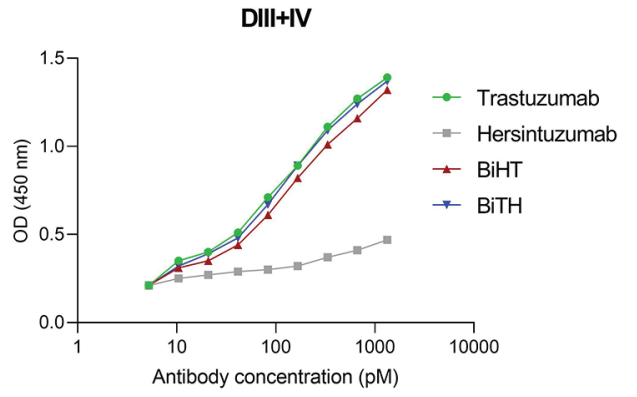

FIGURE 2 | The profile of binding of DVD-Igs and their parental mAbs to HER2 and its subdomains. Different concentrations of antibodies were comparatively determined by an immunoglobulin-specific ELISA (A). Binding patterns of trastuzumab, hersintuzumab, BiHT, and BiTH with full HER2-ECD and its subdomains DI+II and DIII+IV were analyzed by a specific ELISA (B-D).

TABLE 1 | Affinity constant of bispecific mAbs and the parental antibodies to full HER2-ECD and its subdomains.

\begin{tabular}{lccc}
\hline Antibodies & $\mathbf{K}_{\mathbf{D}}$ (DI+II) $\mathbf{n M}$ & $\mathbf{K}_{\mathbf{D}}$ (DIII+IV) $\mathbf{n M}$ & $\mathbf{K}_{\mathbf{D}}$ (ECD) $\mathbf{n M}$ \\
\hline Trastuzumab & - & 0.59 & 0.84 \\
Hersintuzumab & 0.13 & - & 0.22 \\
BiHT & 0.43 & 1.37 & 0.61 \\
BiTH & 1.41 & 0.38 & 0.50 \\
\hline
\end{tabular}

\section{Effects of Bispecific Antibodies on Tumor Cells Proliferation}

${ }^{3} \mathrm{H}$-thymidine incorporation assay was used to assess the antiproliferative effect of DVD-Igs on BT-474, SKOV-3, NCIN87, and the trastuzumab resistant cell lines, HCC-1954 and JIMT-1. Trastuzumab, hersintuzumab, and their combinations were used as controls. The results indicated that both anti-HER2 DVD-Igs and the combination of trastuzumab and hersintuzumab inhibited the proliferation of BT-474, SKOV-3, and NCI-N87 more significantly than each parental mAb alone, at all treatment doses (Figure 5 and Supplementary Table 1). Interestingly, at lower doses, the antiproliferative effect of BiHT was more potent than BiTH as well as the combination of trastuzumab and hersintuzumab in SKOV-3, NCI-N87, and BT-474 cell lines. Similar results were obtained with the trastuzumab resistant cell line HCC-1954. At higher doses, the antiproliferative effect of $\mathrm{BiHT}$ was as potent as the combination of trastuzumab and hersintuzumab in all cell lines with the exception of BT-474, in which the combination was more potent than BiHT and BiTH (Figure 5 and Supplementary Table 1). However, JIMT-1, the other trastuzumab resistant cell line, displayed a different profile, and the antibodies showed poor antiproliferative effect on this cell line.

\section{Antibody-Dependent Cell-Mediated Cytotoxicity (ADCC)}

LDH release assay was performed using normal human PBMCs as effector cells and SKOV-3 tumor cells as target cells. The results showed that $\mathrm{ADCC}$ activity of $\mathrm{BiHT}$ was comparable to those of trastuzumab and hersintuzumab. However, ADCC activity of BiTH was decreased to a quarter of the parental mAbs (Figure 6). According to the results of the proliferation and ADCC assays, we decided to select BiHT for the rest of the experiments.

\section{Pharmacokinetics Analysis}

In order to study the pharmacokinetics of BiHT, trastuzumab, and hersintuzumab, 12 female mice (three in each group) were administered intraperitoneally (IP) with BiHT, trastuzumab, and hersintuzumab and intravenously with trastuzumab alone. The concentration of each antibody was measured in serum at different time intervals by a sandwich ELISA. As demonstrated 
A

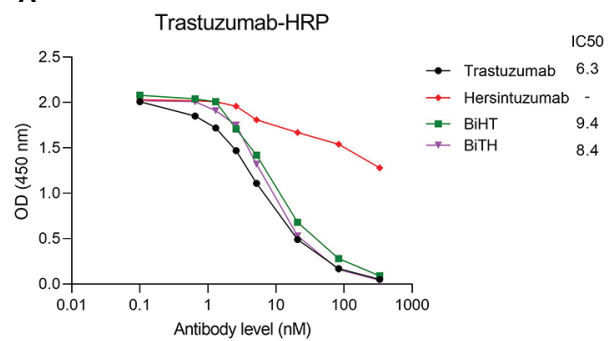

C

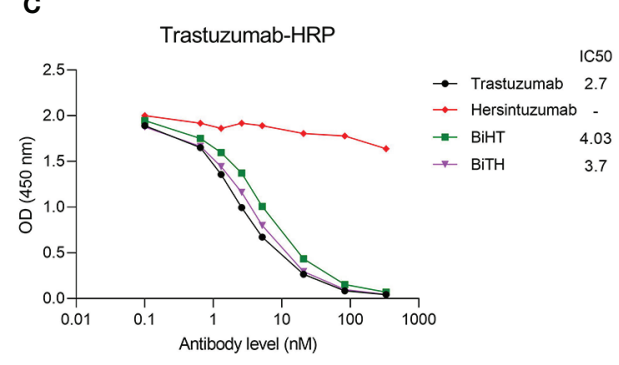

E

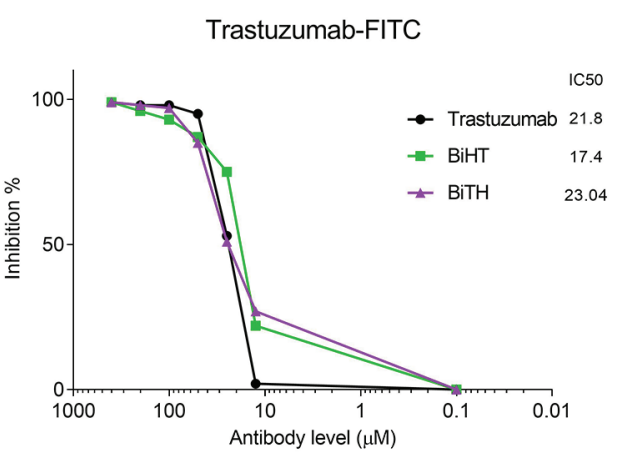

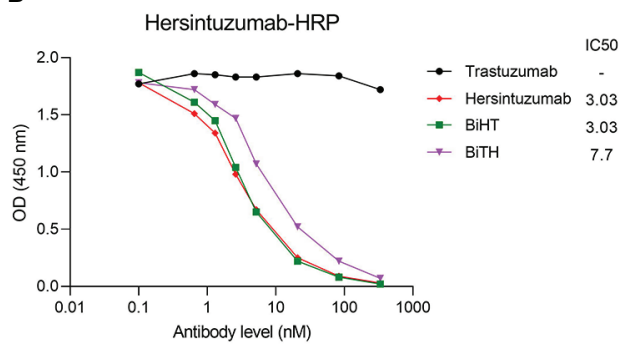

D

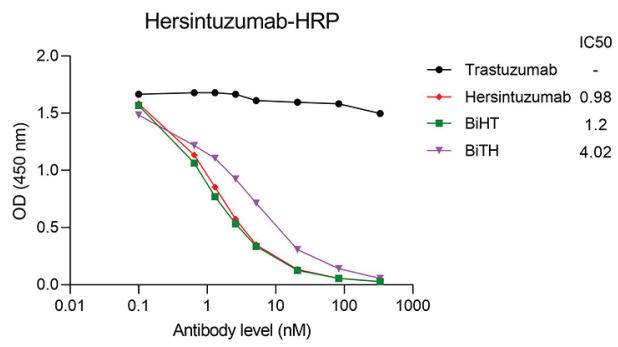

$\mathbf{F}$

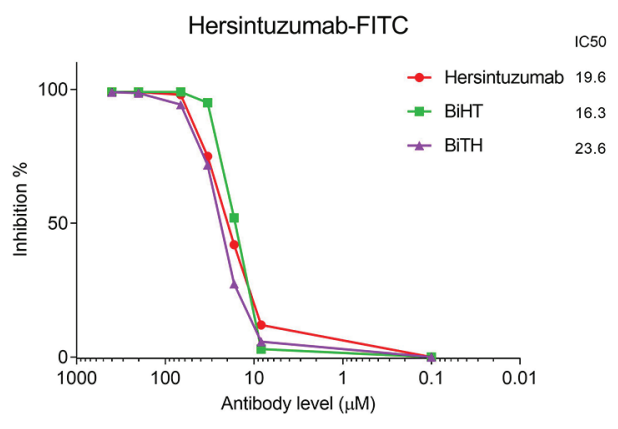

FIGURE 3 | Binding competition and related IC50 of BsAbs. Trastuzumab, hersintuzumab, BiHT, and BiTH compete with trastuzumab-HRP (A, C) or hersintuzumab-HRP (B, D) for binding to recombinant HER2-ECD (A, B), and subdomains III+IV (C) and I+II (D) by ELISA. Binding competition of trastuzumab, hersintuzumab, BiHT, and BiTH with FITC-labeled trastuzumab (E) and FITC-labeled hersintuzumab (F) to native HER2 on SKOV-3 cell line was also assessed by flow cytometry. IC50: The half maximal inhibitory concentration.

in Figure 7 and Table 2, the pharmacokinetic parameters of $\mathrm{BiHT}$ were similar to those of the parental antibodies, indicating that this $\mathrm{BsAb}$ is probably stable in vivo, although stability needs to be confirmed with other experiments such as Western blotting. In order to substantiate our pharmacokinetic results and compare them with the results reported in the literature, since most papers have employed a single IV administration of trastuzumab, we decided to include a single IV administration of trastuzumab in our study. Our data are compatible with those reported by other investigators (14). Since cross reactivity of the anti-human HER2 mAbs with murine HER2 affects the pharmacokinetics of these mAbs, we checked cross reactivity of hersintuzumab and transtuzumab with mouse HER2 by flow cytometry, using a HER2 expressing mouse cell line (3T3-L1). No cross reactivity was observed (Supplementary Figure 1).

\section{Inhibition of Cell Signaling and HER2 Downregulation}

MAPK and AKT/PI3K are the two major signaling pathways affected by the HER family. We evaluated the effects of BiHT and trastuzumab plus hersintuzumab on the phosphorylation of these signaling pathways in BT-474, SKOV-3, NCI-N87, HCC1954, JIMT-1, and MCF-7 cell lines. The results showed that BiHT induced almost complete inhibition of phosphorylation of AKT and ERK1/2 in BT-474, SKOV-3, and NCI-N87, while it slightly suppressed phosphorylation of AKT and ERK in HCC1954 and ERK in MCF-7, but failed to inhibit AKT/ERK signaling pathways in JIMT-1 cell line. All these inhibitory effects were similar to those obtained by the combination of parental antibodies, with the exception of BT-474 and NCI-N87, which displayed more potent phosphorylation inhibition of 


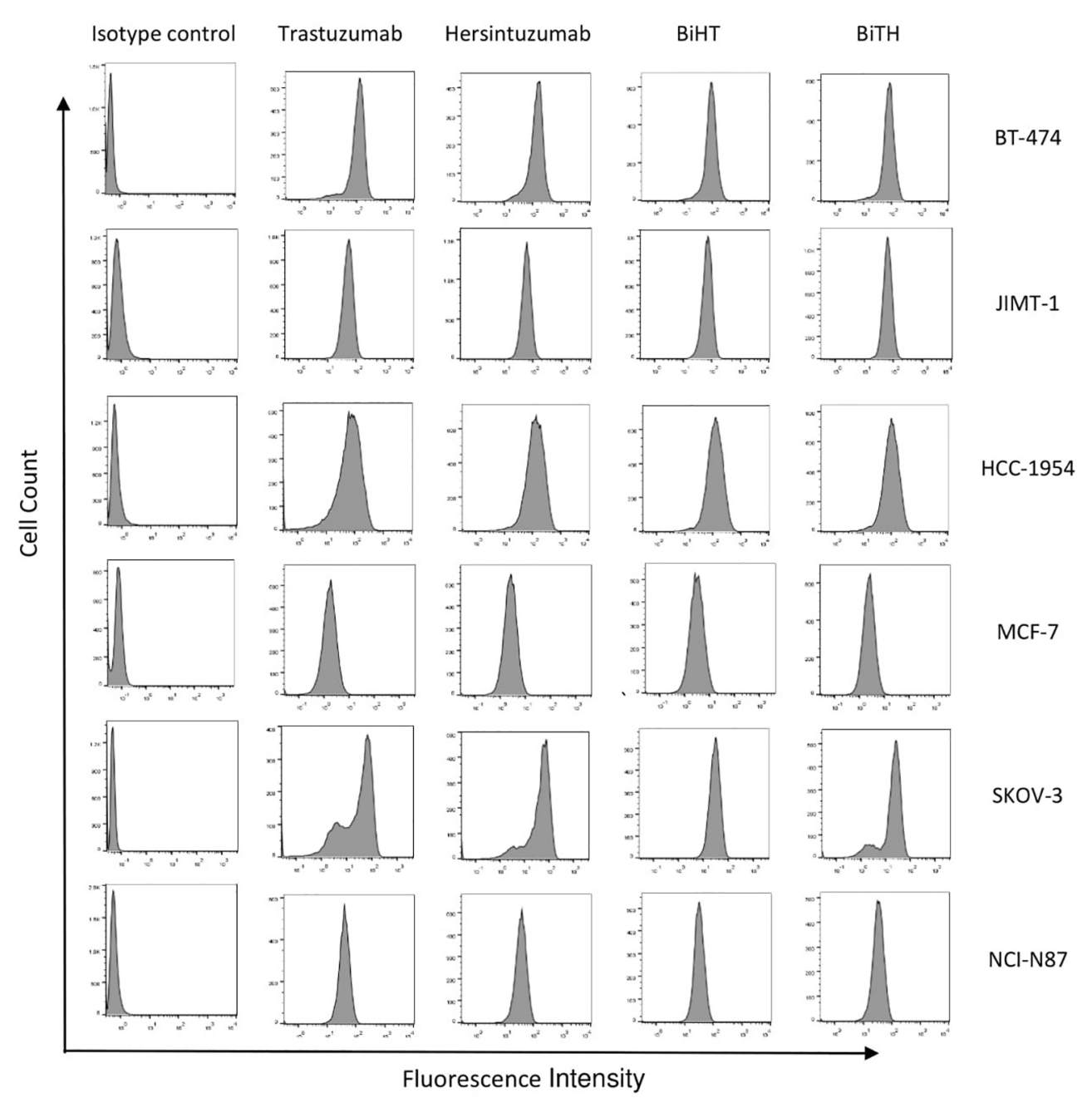

FIGURE 4 | Binding profile of DVD-Igs and their parental antibodies to HER2-expressing tumor cell lines. BT-474, JIMT-1, HCC-1954, MCF-7, SKOV-3, and NCIN87 cell lines were treated with trastuzumab, hersintuzumab, BiHT, BiTH as primary antibodies and then FITC labeled anti-human lg polyclonal antibody was used as the secondary antibody. The final results were analyzed by flow cytometry. A chimeric anti-hepatitis B mAb harboring human lgG1/ $\mathrm{k}$ was used as isotype control.

AKT/ERK by BiHT than the combination of trastuzumab and hersintuzumab. The combination therapy was slightly more potent than BiHT in the phosphorylation inhibition of AKT in SKOV-3 (Figure 8). Interestingly, BiHT downregulated HER2 expression more efficiently than the combination of the two parental mAbs in almost all cell lines, except for JIMT-1 and HCC-1954.

\section{Growth Suppression of Ovarian and Gastric Cancer Xenografts in Nude Mice}

The potential therapeutic efficacy of BiHT and the combination of trastuzumab and hersintuzumab was determined in nude $\mathrm{BALB} / \mathrm{c}$ mice bearing established SKOV-3 and NCI-N87 xenografts. The results in SKOV-3 xenograft indicate that mice treated with BiHT and the combination of the two parental mAbs had significantly lower tumor size than the control group (excipient). Mice treated with the combination of mAbs displayed relatively smaller tumor sizes than the BiHT group, but the differences were statistically insignificant (Figure 9). Similar results were obtained in NCI-N87 xenograft treated mice. Tumor sizes of mice treated with BiHT and the combination of mAbs were highly similar to each other and significantly lower than those of the control group (Figure 9). Moreover, we have previously shown in a pilot study that the combination of trastuzumab and hersituzumab was more potent than each antibody alone for regression of both NCI-N87 and SKOV-3 xenograft tumors (Supplementary Figure 2).

\section{DISCUSSION}

Despite the great success of mAbs in cancer therapy, many efforts are being made to improve their therapeutic efficacy. Combination therapy of two mAbs is a promising strategy to achieve synergistic 

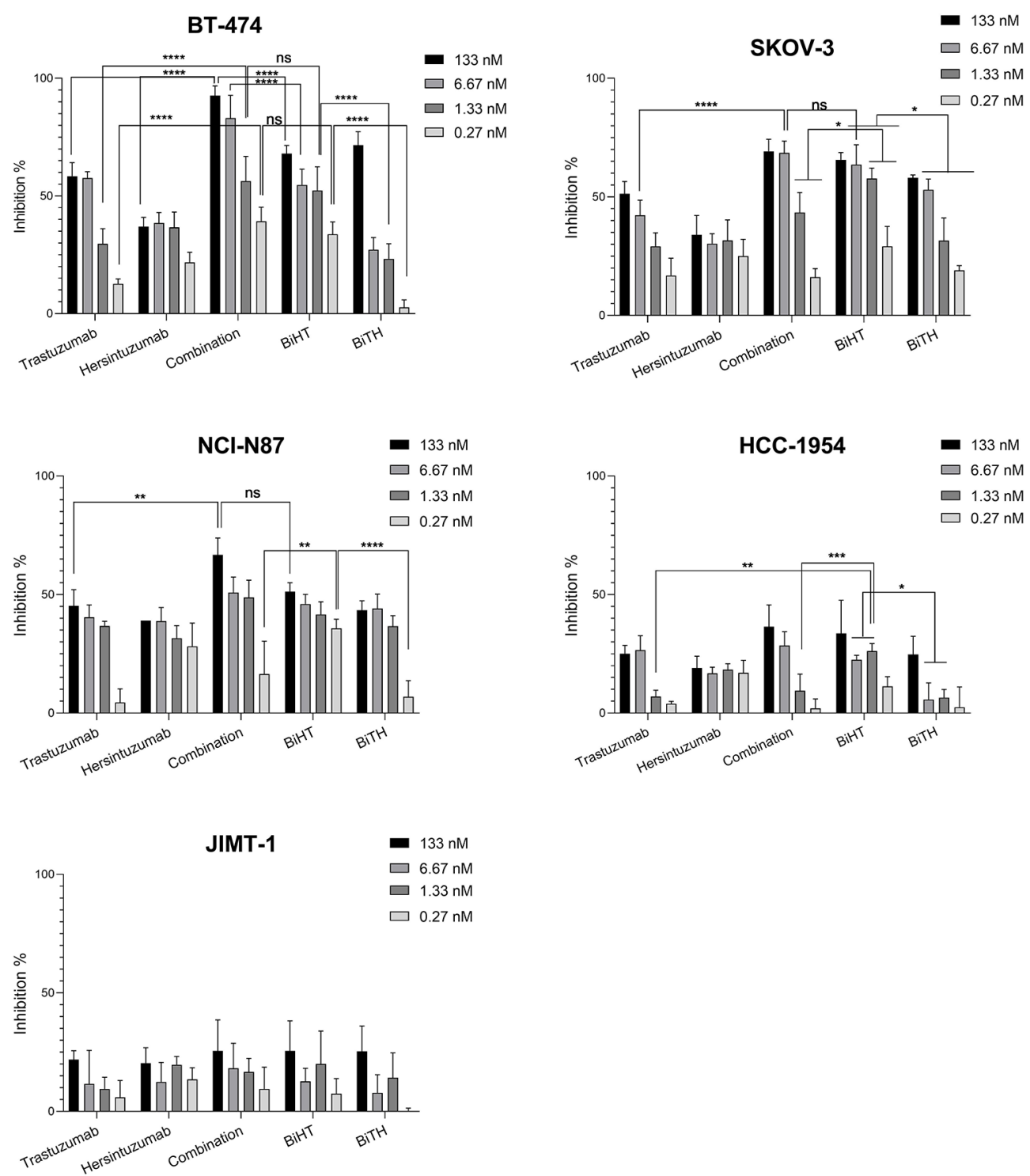

FIGURE 5 | Anti-proliferative effects of BsAbs and their parental mAbs on the proliferation of BT-474, HCC-1954, JIMT-1, SKOV-3, and NCI-N87 cell lines. Data represent percentage of cell growth inhibition of triplicate cultures conducted three times. Combination denotes treatment with trastuzumab and hersintuzumab at a half dose of the indicated concentration for each mAbs. ${ }^{\star \star \star \star} \mathrm{P}<0.0001$, ${ }^{\star \star \star} \mathrm{P}<0.001,{ }^{\star \star} \mathrm{P}<0.01$, ${ }^{\star} \mathrm{P}<0.05$, ns: non-significant.

effects of anti-cancer therapy. Combination of trastuzumab and pertuzumab disrupted the heterodimerization of HER2 with HER3, inhibited AKT signaling in BT-474, and suppressed HER2-positive breast and non-small cell lung cancer xenografts more potently than either monotherapy $(49,50)$. Many research groups try to develop novel anti-HER2 mAbs hoping to use them in combination with another anti-HER2 $\mathrm{mAb}$ to obtain better inhibitory synergistic effects (7-9).

In our previous work, we developed a new humanized mAb designated hersintuzumab, which recognizes different epitope than trastuzumab and pertuzumab without any cross-reactivity with other members of the HER family (US patent: US20170066829A1). Preliminary functional characterization of this $\mathrm{mAb}$ indicated that it induces cell cycle arrest at G1 phase and in combination with trastuzumab had more potent antiproliferative effect on tumor cell lines than the combination of pertuzumab and trastuzumab (12). Moreover, its combination with trastuzumab significantly inhibited both AKT and ERK phosphorylation more potently than the combination of trastuzumab and pertuzumab (48). In accordance with in vitro studies, hersintuzumab in combination with trastuzumab showed superior anti-tumor activity than each $\mathrm{mAb}$ alone in ovarian and gastric xenograft bearing mice (Supplementary Figure 2), which makes it a perfect partner for generation of a new BsAb antibody.

Converting non-overlapping and non-competitive mAbs to a $\mathrm{Bs} \mathrm{Ab}$ antibody enables us to have synergistic effects of two mAbs 


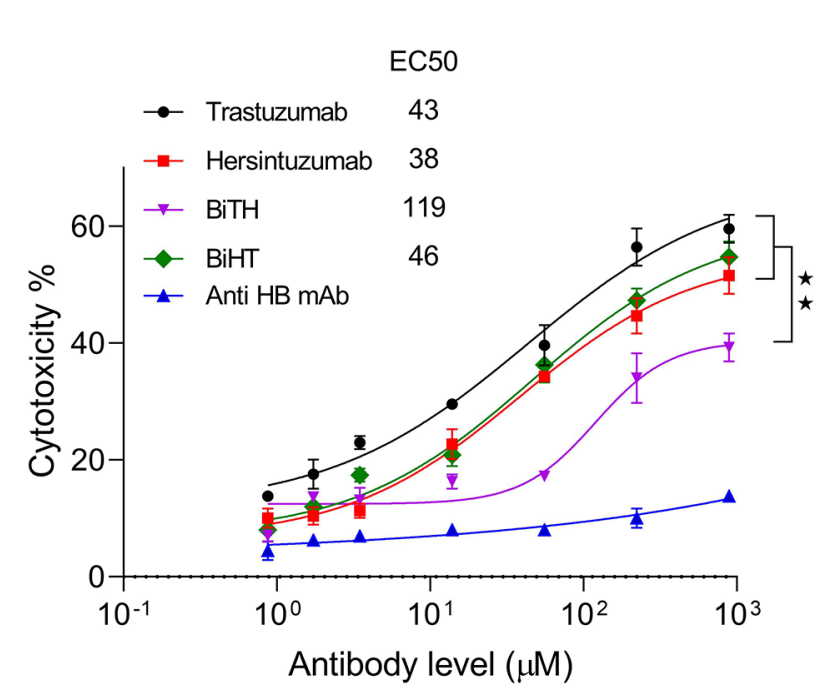

FIGURE 6 | Antibody dependent cell cytotoxicity (ADCC) of the BsAbs. SKOV-3 cells were cocultured with human PBMCs in the prescence of serial concentrations of $\mathrm{mAbs}$ and $\mathrm{LDH}$ release was measured in the supernatants. A chimeric anti-hepatitis B mAb harboring human $\lg \mathrm{G} 1 / \kappa$ was used as isotype negative control. The ADCC experiments were performed in triplicate, and the data are representative of mean \pm SEM of two independent experiments. EC50: The half maximal effective concentration, HB: hepatitis B, ${ }^{* \star} \mathrm{P}<0.01$

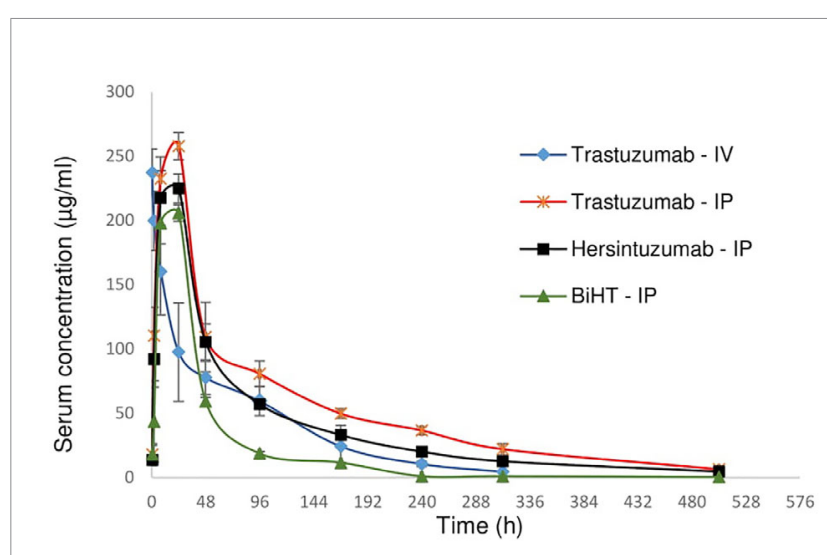

FIGURE 7 | Pharmacokinetics profile of BiHT, trastuzumab, and hersintuzumab. Serum concentrations $( \pm$ SEM) were measured at different time intervals after administration of a single dose of $10 \mathrm{mg} / \mathrm{kg}$ (intraperitoneally/IP or intravenously/IV) in BALB/c mice (3 mice/group). IP: intraperitoneal, IV: intravenous.

in one molecule (30). BsAb antibodies that bind two different epitopes may have synergistic effects on different aspects of HER2 functions, such as heterodimerization and cell signaling. At least three research groups developed anti-HER2 BsAbs from trastuzumab and pertuzumab in different formats $(14,16,17)$. In this study, we used DVD-Ig technology (31) to develop two BsAbs, designated $\mathrm{BiHT}$ and $\mathrm{BiTH}$, using variable domains of trastuzumab and our new anti-HER2 mAb, hersintuzumab, which bind to the domain IV and I+II of human HER2-ECD, respectively. We used a long linker between variable domains of trastuzumab and hersintuzumab in the light and heavy chains of $\mathrm{BiHT}$ and BiTH. The length of the linkers between two tandem variable domains of a DVD-Ig has been shown to affect the affinity of the inner domain, long linkers display better flexibility and binding affinity than short linkers for some variables domain of mAbs $(44,45,51,52)$. Another consideration in the design of DVD-Ig is the orientation of the two variable domains (53). Accordingly, we generated $\mathrm{BiHT}$ and $\mathrm{BiTH}$, in which hersintuzumab is in the outer and trastuzumab is in the inner variable domain and vice versa, respectively.

Both DVD-Igs were transfected and expressed in $\mathrm{CHO}-\mathrm{K} 1$ cells without any intermediate light or heavy chain surplus byproduct after purification and they retained their IgG like structure (Figure 1B). We found that the binding affinity of trastuzumab variable domain in BiHT and BiTH is similar to the corresponding parental $\mathrm{mAb}$, trastuzumab. Moreover, binding affinity of hersintuzumab variable domain in BiTH is far less than that of BiHT (Table 1). In fact, when trastuzumab variable domain is in the outer or inner domain of DVD-Ig, its reactivity doesn't change profoundly. In contrast, the reactivity of hersintuzumab variable domain decreases in the inner, but not in the outer position. In accordance with our results, $\mathrm{Wu}$ and colleague showed that IL-1a variable domain retains potency of parental $\mathrm{mAb}$, even with short linker, in the inner and outer domains of DVD-Ig. In contrast, potency of IL-1b variable domain reduces in the inner domain of DVD-Ig and it seems that IL-1a variable domain is more compatible with the inner domain position than IL-1b variable domain (44). Bohua et al. showed that with the use of a long linker, trastuzumab keeps its potency either in the inner or outer layer of DVD-Igs, but not with a short linker (14). In another study it was demonstrated that trastuzumab retains its activity in both orientations, even with a short linker (16).

The results obtained from ELISA and flow cytometry (Figures 3C, D) indicated that despite the decrease in the binding activity of BiTH to subdomain I+II (Figure 2C), there is no significant difference between $\mathrm{BiHT}$ and $\mathrm{BiTH}$ for binding to recombinant full HER2-ECD and native HER2 on the surface of different cell lines (Table 1 and Figure 4), which might be due to the compensatory binding activity of the variable domain of trastuzumab. None of the previous works used subdomains of HER2 to determine the reactivity and affinity of their BsAbs $(14,16)$.

Antiproliferative effect of BiHT on different HER2overxpressing cell lines showed that with the exception of the JIMT-1 cell line, it inhibits the growth of all cell lines doses dependently, similar to the combination of the parental mAbs. At subsaturated doses, BiHT showed more potent growth inhibitory effects than the combination of the two parental mAbs or BiTH. In contrast to BiTH, the ADCC activity of $\mathrm{BiHT}$ was similar to the parental mAbs. Significantly lower ADCC activity of BiTH compared to BiHT could be associated with its lower HER2 binding affinity. The effect of antigen binding affinity on ADCC activity has already been reported for other antibody specificities $(17,54,55)$. As a result, we omitted BiTH from the rest of the experiments and focused on 
TABLE 2 | Pharmacokinetic parameters of BiHT, trastuzumab, and hersintuzumab in BALB/c mice (3 mice/group).

\begin{tabular}{|c|c|c|c|c|}
\hline & & Trastuzumab & Hersintuzumab & BiHT \\
\hline \multirow[t]{4}{*}{ IP } & $\operatorname{Tmax}(\mathrm{h})$ & 24 & 24 & 24 \\
\hline & Cmax ( $\mu \mathrm{g} / \mathrm{ml})$ & $258 \pm 18$ & $225 \pm 24$ & $206 \pm 2$ \\
\hline & $\mathrm{T} 1 / 2$ (h) & $124.8 \pm 7$ & $105 \pm 6$ & $82 \pm 4$ \\
\hline & Clearance (L/h) & 0.004 & 0.005 & 0.005 \\
\hline \multirow[t]{3}{*}{ IV } & & & Trastuzumab & \\
\hline & Clearance (L/h) & & 0.006 & \\
\hline & $\mathrm{T} 1 / 2(\mathrm{~h})$ & & $119.04 \pm 5$ & \\
\hline
\end{tabular}

Tmax, time point of maximum concentration of the antibodies; Cmax, maximum concentration of the antibodies; T1/2, half-life; IP, intraperitoneal; IV, intravenous.

BiHT. The growth inhibitory results were in accordance with the results of downstream signaling pathways, showing significant inhibitory of phosphorylation of AKT and ERK by BiHT treatment (Figure 8). Jinming $\mathrm{Gu}$ et al. developed eight antiHER2 DVD-Igs from trastuzumab and pertuzumab with different orientations of variable domains and different linker sizes. Four of these DVD-Igs inhibited the cell proliferation of NCI-N87 cells while the other four DVD-Igs enhanced the proliferation of this cell line (16). Enhancement or inhibition of cell proliferation by these two groups of BsAb was attributed to the avidity, together with domain orientation of DVD-Igs, which may induce different HER2 conformational changes. Both of our DVD-Igs displayed anti-proliferative effects in four cell lines from three different tissue origins.

In ovarian and gastric cancer cell lines, SKOV-3 and NCIN87, BiHT suppressed xenograft tumor growth as efficiently as the combination of trastuzumab and hersintuzumab (Figure 9). In our pilot study we observed that the combination of trastuzumab and hersintuzumab was significantly more effective than each $\mathrm{mAb}$ alone for the inhibition of SKOV-3 and NCI-N87 xenograft models (Supplementary Figure 2).

As we can see from the proliferation experiments of SKOV-3 and NCI-N87 cell lines (Figure 5), there is little difference between 1 and $20 \mu \mathrm{g} / \mathrm{ml}$ of $\mathrm{mAb}$ concentration for proliferation inhibition, which indicates that $1 \mu \mathrm{g} / \mathrm{ml}$ is a saturated concentration. Moreover, in the pharmacokinetics experiments, serum concentration of all administrated mAbs is more than $1 \mu \mathrm{g} / \mathrm{ml}$ one week after $10 \mathrm{mg} / \mathrm{kg}$ IP or IV administration (Figure 7). Based on these considerations we decided to use $10 \mathrm{mg} / \mathrm{kg}$ of the combination of trastuzumab and hersintuzumab or BiHT once a week in our xenograft model. Harumi Sakahara et al. demonstrated that the concentration of the antibodies in the tissues expressing the specific ligand is $1.2-17$ times more than
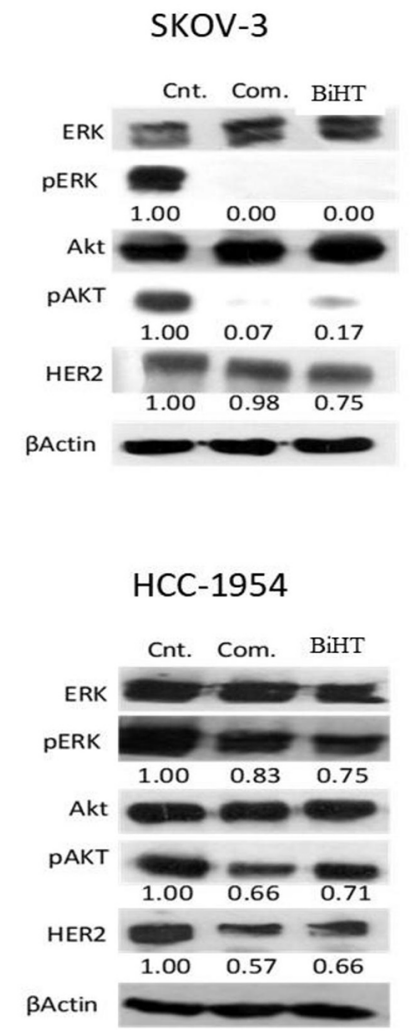

BT-474

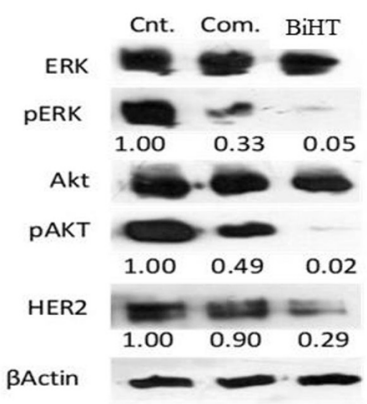

JIMT-1

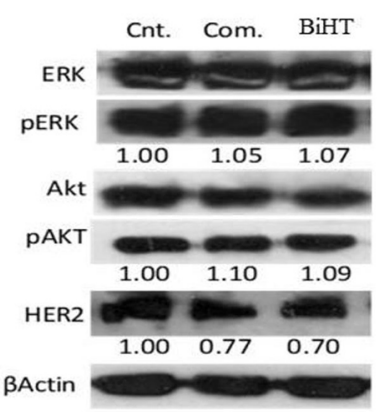

$\mathrm{NCl}-\mathrm{N} 87$

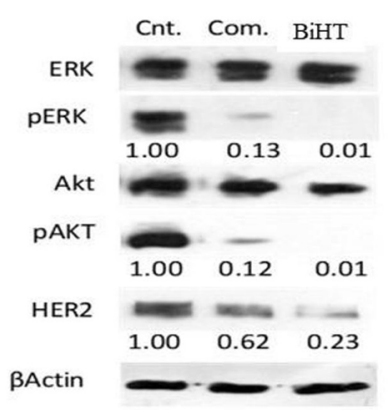

MCF-7

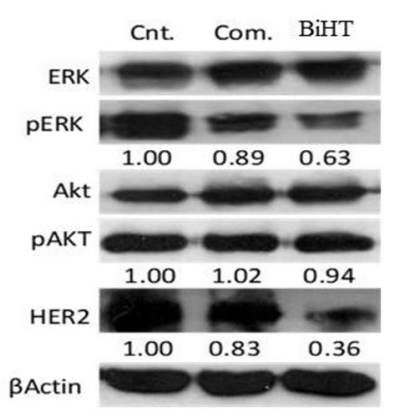

FIGURE 8 | Inhibitory effects of BiHT on phosphorylation of the AKT and ERK1/2 signaling pathways and HER2 expression. BT-474, SKOV-3, NCI-N87, HCC1954, JIMT-1, and MCF-7 cell lines were treated with BiHT $(20 \mu \mathrm{g} / \mathrm{ml})$ or combination of trastuzumab and hersintuzumab $(10 \mu \mathrm{g} / \mathrm{ml}$, each) for $24 \mathrm{~h}$. Cell lysates were separated on SDS-PAGE and immunoblotted to detect AKT, pAKT, ERK1/2, pERK1/2, HER2, and beta actin as a house keeping protein. Cnt: Control, Com: Combination. The figures presented underneath of each mAb treatment represent the band densities calculated as described in Materials and Methods. 


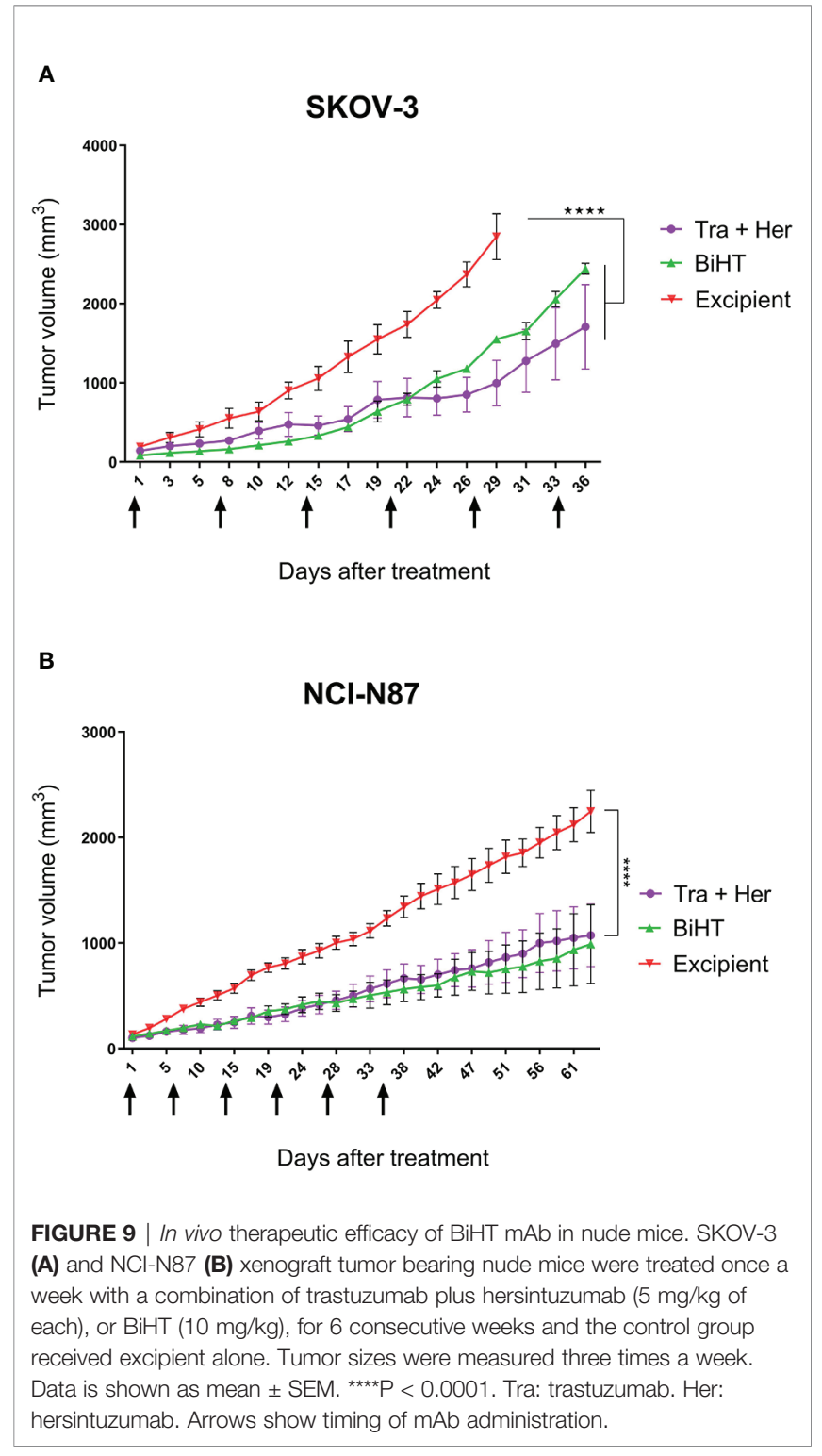

their serum concentration after 96 hours of injection (56). As a consequence, it could be deduced that the dose of the antibodies in the tumor niche of our xenograft model is much more than the saturation dose and similar to the proliferation experiments, there is no difference between BiHT and the combination of trastuzumab and hersintuzumab at this dose.

Altogether, our results demonstrated that BiHT was as efficient as the combination of the parental mAbs in vitro and in vivo. As a result, having the additive or synergistic effects of the two $\mathrm{mAbs}$ in one BsAb reduces drug cost due to manufacturing of a single mAb instead of two mAbs, improves safety of patients due to less manipulation, and increases patients' satisfaction.

\section{CONCLUSION}

In this study we demonstrated that our novel BsAb (BiHT), designated trasintuzumab, is a dual functional and in vivo stable
IgG1 antibody, which inhibits cell proliferation, downregulates HER2 expression, and blocks AKT and ERK downstream signaling pathways. It displayed anti-tumor activity in vitro and in vivo as efficiently as the combination of the parental mAbs trastuzumab and hersintuzumab. Trasintuzumab is expected to exhibit more potent therapeutic effects than the combination of trastuzumab and pertuzumab and is a potential therapeutic candidate for treatment of HER2 overexpressing cancer types.

\section{DATA AVAILABILITY STATEMENT}

The raw data supporting the conclusions of this article will be made available by the authors, without undue reservation.

\section{ETHICS STATEMENT}

The animal study was reviewed and approved by ethics committee of National Institute for Medical Research Development of Iran - NIMAD (IR NIMAD REC 1396 060).

\section{AUTHOR CONTRIBUTIONS}

FS and MMA made substantial contributions to the study conception, design, analysis and interpretation of the data. MM made substantial contributions to the design, acquisition, analysis and interpretation of the data. MA, TB, HZ, FH, FS, MAJ and MM contributed to the acquisition of the data. The first draft of the manuscript was written by MM and MMA. FS, FG-S and MJ-T commented on subsequent versions of the manuscript. All authors contributed to the article and approved the submitted version. FS is the guarantor for the overall content of the article.

\section{FUNDING}

This study was partially supported by grants from National Institute for Medical Research Development of Iran (Grant No. 957625), Tehran University of Medical Sciences (Grant No. $97-$ 02-27-38122), Avicenna Research Institute (Grant No. 97-004), and Academic Center for Education, Culture and Research/ ACECR (Grant No. 3075-33).

\section{ACKNOWLEDGMENTS}

The authors wish to express their thanks to Prof. A. H. Zarnani for scientific consultation and Jalal Khoshnoodi for technical support.

\section{SUPPLEMENTARY MATERIAL}

The Supplementary Material for this article can be found online at: https://www.frontiersin.org/articles/10.3389/fimmu.2020. 600883/full\#supplementary-material 


\section{REFERENCES}

1. Graus-Porta D, Beerli RR, Daly JM, Hynes NE. ErbB-2, the preferred heterodimerization partner of all ErbB receptors, is a mediator of lateral signaling. EMBO J (1997) 16(7):1647-55. doi: 10.1093/emboj/16.7.1647

2. Citri A, Yarden Y. EGF-ERBB signalling: towards the systems level. Nat Rev Mol Cell Biol (2006) 7(7):505-16. doi: 10.1038/nrm1962

3. Tai W, Mahato R, Cheng K. The role of HER2 in cancer therapy and targeted drug delivery. J Controlled Release (2010) 146(3):264-75. doi: 10.1016/ j.jconrel.2010.04.009

4. Hudis CA. Trastuzumab-mechanism of action and use in clinical practice. $N$ Engl J Med (2007) 357(1):39-51. doi: 10.1056/NEJMra043186

5. Adams CW, Allison DE, Flagella K, Presta L, Clarke J, Dybdal N, et al. Humanization of a recombinant monoclonal antibody to produce a therapeutic HER dimerization inhibitor, pertuzumab. Cancer Immunology Immunotherapy (2005) 55(6):717. doi: 10.1007/s00262-005-0058-x

6. Baselga J, Cortes J, Kim SB, Im SA, Hegg R, Im YH, et al. Pertuzumab plus trastuzumab plus docetaxel for metastatic breast cancer. N Engl J Med (2012) 366(2):109-19. doi: 10.1056/NEJMoa1113216

7. Ko B-K, Lee S-Y, Lee Y-H, Hwang I-S, Persson H, Rockberg J, et al. Combination of novel HER2-targeting antibody 1E11 with trastuzumab shows synergistic antitumor activity in HER2-positive gastric cancer. Mol Oncol (2015) 9(2):398-408. doi: 10.1016/j.molonc.2014.09.007

8. Pedersen MW, Jacobsen HJ, Koefoed K, Dahlman A, Kjær I, Poulsen TT, et al. Targeting three distinct HER2 domains with a recombinant antibody mixture overcomes trastuzumab resistance. Mol Cancer Ther (2015) 14(3):669-80. doi: 10.1158/1535-7163.MCT-14-0697

9. Meng Y, Zheng L, Yang Y, Wang H, Dong J, Wang C, et al. A monoclonal antibody targeting ErbB2 domain III inhibits ErbB2 signaling and suppresses the growth of ErbB2-overexpressing breast tumors. Oncogenesis (2016) 5: e211. doi: 10.1038/oncsis.2016.25

10. Shu M, Yan H, Xu C, Wu Y, Chi Z, Nian W, et al. A novel anti-HER2 antibody GB235 reverses Trastuzumab resistance in HER2-expressing tumor cells in vitro and in vivo. Sci Rep (2020) 10(1):e2986. doi: 10.1038/s41598-020-59818-2

11. Tahmasebi F, Kazemi T, Amiri MM, Khoshnoodi J, Mahmoudian J, Bayat AA, et al. In vitro assessment of the effects of anti-HER2 monoclonal antibodies on proliferation of HER2-overexpressing breast cancer cells. Immunotherapy (2013) 6(1):43-9. doi: 10.2217/imt.13.156

12. Amiri MM, Golsaz-Shirazi F, Soltantoyeh T, Hosseini-Ghatar R, Bahadori T, Khoshnoodi J, et al. Hersintuzumab: A novel humanized anti-HER2 monoclonal antibody induces potent tumor growth inhibition. Invest New Drugs (2018) 36(2):171-86. doi: 10.1007/s10637-017-0518-0

13. Labrijn AF, Janmaat ML, Reichert JM, Parren PWHI. Bispecific antibodies: a mechanistic review of the pipeline. Nat Rev Drug Discov (2019) 18(8):585608. doi: 10.1038/s41573-019-0028-1

14. Li B, Meng Y, Zheng L, Zhang X, Tong Q, Tan W, et al. Bispecific antibody to ErbB2 overcomes trastuzumab resistance through comprehensive blockade of ErbB2 heterodimerization. Cancer Res (2013) 73(21):6471-83. doi: 10.1158/ 0008-5472.can-13-0657

15. Brack S, Attinger-Toller I, Schade B, Mourlane F, Klupsch K, Woods R, et al. A bispecific HER2-targeting FynomAb with superior antitumor activity and novel mode of action. Mol Cancer Ther (2014) 13(8):2030-9. doi: 10.1158/ 1535-7163.mct-14-0046-t

16. Gu J, Yang J, Chang Q, Lu X, Wang J, Chen M, et al. Identification of AntiErbB2 Dual Variable Domain Immunoglobulin (DVD- $\mathrm{Ig}^{\mathrm{TM}}$ ) Proteins with Unique Activities. PLoS One (2014) 9(5):e97292. doi: 10.1371/ journal.pone.0097292

17. Zhang F, Zhang J, Liu M, Zhao L, LingHu R, Feng F, et al. Combating HER2overexpressing breast cancer through induction of calreticulin exposure by Tras-Permut CrossMab. Oncoimmunology (2015) 4(3):e994391. doi: 10.4161/ 2162402x.2014.994391

18. Li JY, Perry SR, Muniz-Medina V, Wang X, Wetzel LK, Rebelatto MC, et al. A biparatopic HER2-targeting antibody-drug conjugate induces tumor regression in primary models refractory to or ineligible for HER2-targeted therapy. Cancer Cell (2016) 29(1):117-29. doi: 10.1016/j.ccell.2015.12.008

19. Zhang Y, Wang L, Chong X, Yu X, Meng Y, Dong J, et al. A bispecific antiErbB2 antibody potently induces ErbB2 internalization and suppresses
ErbB2-overexpressing tumor growth. Biochem Biophys Res Commun (2016) 477(4):755-60. doi: 10.1016/j.bbrc.2016.06.131

20. Liu J, Wu X, Lin L, Pan H, Wang Y, Li Y, et al. Bp-Bs, a novel T-cell engaging bispecific antibody with biparatopic Her2 binding, has potent anti-tumor activities. Mol Ther Oncolytics (2019) 14:66-73. doi: 10.1016/ j.omto.2019.03.009

21. McDonagh CF, Huhalov A, Harms BD, Adams S, Paragas V, Oyama S, et al. Antitumor activity of a novel bispecific antibody that targets the ErbB2/ErbB3 oncogenic unit and inhibits heregulin-induced activation of ErbB3. Mol Cancer Ther (2012) 11(3):582-94. doi: 10.1158/1535-7163.MCT-11-0820

22. Malm M, Bass T, Gudmundsdotter L, Lord M, Frejd FY, Stahl S, et al. Engineering of a bispecific affibody molecule towards HER2 and HER3 by addition of an albumin-binding domain allows for affinity purification and in vivo half-life extension. Biotechnol J (2014) 9(9):1215-22. doi: 10.1002/ biot.201400009

23. Diermeier-Daucher S, Ortmann O, Buchholz S, Brockhoff G. Trifunctional antibody ertumaxomab: Non-immunological effects on Her2 receptor activity and downstream signaling. MAbs (2012) 4(5):614-22. doi: 10.4161/ mabs. 21003

24. Vaishampayan U, Thakur A, Rathore R, Kouttab N, Lum LG. Phase I study of anti-CD3 x Anti-Her2 bispecific antibody in metastatic castrate resistant prostate cancer patients. Prostate Cancer (2015) 2015:e285193. doi: 10.1155/ 2015/285193

25. Kiewe P, Hasmüller S, Kahlert S, Heinrigs M, Rack B, Marmé A, et al. Phase I trial of the trifunctional anti-HER2 $\times$ anti-CD3 antibody ertumaxomab in metastatic breast cancer. Clin Cancer Res (2016) 12(10):3085-91. doi: 10.1186/ s12885-016-2449-0

26. Yu S, Zhang J, Yan Y, Yao X, Fang L, Xiong H, et al. A novel asymmetrical anti-HER2/CD3 bispecific antibody exhibits potent cytotoxicity for HER2positive tumor cells. J Exp Clin Cancer Res (2019) 38(1):e355. doi: 10.1186/ s13046-019-1354-1

27. Mittal D, Vijayan D, Neijssen J, Kreijtz J, Habraken MMJM, Van Eenennaam $\mathrm{H}$, et al. Blockade of ErbB2 and PD-L1 using a bispecific antibody to improve targeted anti-ErbB2 therapy. Oncoimmunology (2019) 8(11):e1648171. doi: 10.1080/2162402X.2019.1648171

28. Hinner MJ, Aiba RSB, Jaquin TJ, Berger S, Dürr MC, Schlosser C, et al. Tumor-localized costimulatory T-cell engagement by the 4-1BB/HER2 bispecific antibody-anticalin fusion PRS-343. Clin Cancer Res (2019) 25(19): e5878. doi: 10.1158/1078-0432.CCR-18-3654

29. de Goeij BE, Vink T, Ten Napel H, Breij EC, Satijn D, Wubbolts R, et al. Efficient payload delivery by a bispecific antibody-drug conjugate targeting HER2 asnd CD63. Mol Cancer Ther (2016) 15(11):2688-97. doi: 10.1158/ 1535-7163.mct-16-0364

30. Wu X, Demarest SJ. Building blocks for bispecific and trispecific antibodies. Methods (San Diego Calif). (2019) 154:3-9. doi: 10.1016/j.ymeth.2018.08.010

31. Wu C, Ying H, Grinnell C, Bryant S, Miller R, Clabbers A, et al. Simultaneous targeting of multiple disease mediators by a dual-variable-domain immunoglobulin. Nat Biotechnol (2007) 25(11):1290-7. doi: 10.1038/nbt1345

32. Gu J, Yang J, Chang Q, Liu Z, Ghayur T, Gu J. Identification of anti-EGFR and anti-ErbB3 dual variable domains immunoglobulin (DVD-Ig) proteins with unique activities. PLoS One (2015) 10(5):e0124135. doi: 10.1371/ journal.pone. 0124135

33. Piccione EC, Juarez S, Liu J, Tseng S, Ryan CE, Narayanan C, et al. A bispecific antibody targeting $\mathrm{CD} 47$ and $\mathrm{CD} 20$ selectively binds and eliminates dual antigen expressing lymphoma cells. MAbs (2015) 7(5):946-56. doi: 10.1080/ 19420862.2015 .1062192

34. Zeng J, Liu R, Wang J, Fang Y. A bispecific antibody directly induces lymphoma cell death by simultaneously targeting CD20 and HLA-DR. J Cancer Res Clin Oncol (2015) 141(11):1899-907. doi: 10.1007/s00432-0151949-7

35. Khatri A, Goss S, Jiang P, Mansikka H, Othman AA. Pharmacokinetics of ABT-122, a TNF- $\alpha$ - and IL-17A-targeted dual-variable domain immunoglobulin, in healthy subjects and patients with rheumatoid arthritis: results from three phase I trials. Clin Pharmacokinetics. (2018) 57:613-23. doi: 10.1007/s40262-017-0580-y

36. Kosloski MP, Goss S, Wang SX, Liu J, Loebbert R, Medema JK, et al. Pharmacokinetics and tolerability of a dual variable domain immunoglobulin ABT-981 against IL-1alpha and IL-1beta in healthy 
subjects and patients with osteoarthritis of the knee. J Clin Pharmacol (2016) 56(12):1582-90. doi: 10.1002/jcph.764

37. Ding K, Eaton L, Bowley D, Rieser M, Chang Q, Harris MC, et al. Generation and characterization of ABBV642, a dual variable domain immunoglobulin molecule (DVD-Ig) that potently neutralizes VEGF and PDGF-BB and is designed for the treatment of exudative age-related macular degeneration. MAbs (2017) 9(2):269-84. doi: 10.1080/19420862.2016.1268305

38. Craig RB, Summa CM, Corti M, Pincus SH. Anti-HIV double variable domain immunoglobulins binding both gp41 and gp120 for targeted delivery of immunoconjugates. PLoS One (2012) 7(10):e46778. doi: 10.1371/ journal.pone.0046778

39. McGaraughty S, Davis-Taber RA, Zhu CZ, Cole TB, Nikkel AL, Chhaya M, et al. Targeting anti-TGF-beta therapy to fibrotic kidneys with a dual specificity antibody approach. J Am Soc Nephrol JASN (2017) 26(12):361626. doi: 10.1681/asn.2017010013

40. Karaoglu Hanzatian D, Schwartz A, Gizatullin F, Erickson J, Deng K, Villanueva R, et al. Brain uptake of multivalent and multi-specific DVD-Ig proteins after systemic administration. MAbs (2018) 10(5):765-77. doi: $10.1080 / 19420862.2018 .1465159$

41. Li Y, Hickson JA, Ambrosi DJ, Haasch DL, Foster-Duke KD, Eaton LJ, et al. ABT-165, a dual variable domain immunoglobulin (DVD-Ig) targeting DLL4 and VEGF, demonstrates superior efficacy and favorable safety profiles in preclinical models. Mol Cancer Ther (2018) 17(5):1039-50. doi: 10.1158/15357163.Mct-17-0800

42. Ibrahim S, Girault A, Ohresser M, Lereclus E, Paintaud G, Lecomte T, et al. Monoclonal antibodies targeting the IL-17/IL-17RA axis: an opportunity to improve the efficiency of anti-VEGF therapy in fighting metastatic colorectal cancer. Clin Colorectal Cancer (2018) 17(1):109-13. doi: 10.1016/ j.clcc.2017.10.003

43. Fleischmann RM, Bliddal H, Blanco FJ, Schnitzer TJ, Peterfy C, Chen S, et al. A phase II trial of lutikizumab, an anti-interleukin- $1 \alpha / \beta$ dual variable domain immunoglobulin, in knee osteoarthritis patients with synovitis. Arthritis Rheumatol (Hoboken NJ). (2019) 71(7):1056-69. doi: 10.1002/art.40840

44. Chengbin Wu HY, Bose S, Miller R, Medina L, Ghayur LST. Molecular construction and optimization of anti-human IL- $1 \alpha / \beta$ dual variable domain immunoglobulin (DVD-IgTM) molecules. mAbs (2009) 1(4):339-47. doi: $10.4161 /$ mabs.1.4.8755

45. DiGiammarino EL, Harlan JE, Walter KA, Ladror US, Edalji RP, Hutchins $\mathrm{CW}$, et al. Ligand association rates to the inner-variable-domain of a dualvariable-domain immunoglobulin are significantly impacted by linker design. mAbs (2011) 3(5):487-94. doi: 10.4161/mabs.3.5.16326

46. Hosseini Ghatar R, Soltantoyeh T, Bahadori T, Golara M, Hassannia H, Khosravi Eghbal R, et al. Epitope mapping of human HER2 specific mouse monoclonal antibodies using recombinant extracellular subdomains. Asian Pacific J Cancer Prev APJCP (2017) 18(11):3103-10. doi: 10.22034/apjcp.2017.18.11.3103

47. Golsaz-Shirazi F, Amiri MM, Farid S, Bahadori M, Bohne F, Altstetter S, et al. Construction of a hepatitis B virus neutralizing chimeric monoclonal antibody recognizing escape mutants of the viral surface antigen (HBsAg). Antiviral Res (2017) 144:153-63. doi: 10.1016/j.antiviral.2017.06.013

48. Soltantoyeh T, Bahadori T, Hosseini-Ghatar R, Khoshnoodi J, Roohi A, Mobini M, et al. Differential effects of inhibitory and stimulatory anti-HER2 monoclonal antibodies on AKT/ERK signaling pathways. Asian Pacific J Cancer Prev APJCP (2018) 19(8):2255-62. doi: 10.22034/apjcp.2018.19.8.2255

49. Scheuer W, Friess T, Burtscher H, Bossenmaier B, Endl J, Hasmann M. Strongly enhanced antitumor activity of Trastuzumab and Pertuzumab combination treatment on HER2-positive human xenograft tumor models. Cancer Res (2009) 69(24):9330-6. doi: 10.1158/0008-5472.CAN-08-4597

50. Nahta R, Hung M-C, Esteva FJ. The HER-2-targeting antibodies trastuzumab and pertuzumab synergistically inhibit the survival of breast cancer cells. Cancer Res (2004) 64(7):2343-46. doi: 10.1158/0008-5472.CAN-03-3856

51. Jakob CG, Edalji R, Judge RA, DiGiammarino E, Li Y, Gu J, et al. Structure reveals function of the dual variable domain immunoglobulin (DVD-Ig) molecule. MAbs (2013) 5(3):358-63. doi: 10.4161/mabs.23977

52. Lacy SE, Wu C, Ambrosi DJ, Hsieh C-M, Bose S, Miller R, et al. Generation and characterization of ABT-981, a dual variable domain immunoglobulin (DVD-Ig(TM)) molecule that specifically and potently neutralizes both IL-1 $\alpha$ and IL-1ß. mAbs (2015) 7(3):605-19. doi: 10.1080/19420862.2015.1026501

53. Gu J, Ghayur T. Generation of dual-variable-domain immunoglobulin molecules for dual-specific targeting. Methods Enzymol (2012) 502:25-41. doi: 10.1016/B978-0-12-416039-2.00002-1

54. Tang Y, Lou J, Alpaugh RK, Robinson MK, Marks JD, Weiner LM. Regulation of Antibody-Dependent Cellular Cytotoxicity by IgG Intrinsic and Apparent Affinity for Target Antigen. J Immunol (2007) 179(5):2815. doi: 10.4049/ jimmunol.179.5.2815

55. Velders MP, van Rhijn CM, Oskam E, Fleuren GJ, Warnaar SO, Litvinov SV. The impact of antigen density and antibody affinity on antibody-dependent cellular cytotoxicity: relevance for immunotherapy of carcinomas. Br J Cancer (1998) 78(4):478-83. doi: 10.1038/bjc.1998.518

56. Sakahara H, Endo K, Koizumi M, Nakashima T, Kunimatsu M, Watanabe Y, et al. Relationship between in vitro binding activity and in vivo tumor accumulation of radiolabeled monoclonal antibodies. J Nucl Med (1988) 29 (2):235-40. doi: 10.1000/res\%23test

Conflict of Interest: The authors declare that the research was conducted in the absence of any commercial or financial relationships that could be construed as a potential conflict of interest.

Copyright (๑ 2021 Mohammadi, Jeddi-Tehrani, Golsaz-Shirazi, Arjmand, Bahadori, Judaki, Shiravi, Zare, Haghighat, Mobini, Amiri and Shokri. This is an open-access article distributed under the terms of the Creative Commons Attribution License (CC BY). The use, distribution or reproduction in other forums is permitted, provided the original author(s) and the copyright owner(s) are credited and that the original publication in this journal is cited, in accordance with accepted academic practice. No use, distribution or reproduction is permitted which does not comply with these terms. 